\title{
Multi-core Processors in Cloud Computing using Cloudsim
}

\author{
Dina R. Salem \\ PhD Student of Computer \\ Science \\ Business Technology \\ department \\ Canadian International College
}

\author{
M. Haggag \\ Professor of Computer Science \\ Vice Dean for Education \& \\ Students Affairs \\ Faculty of Computers and \\ Information \\ Helwan University
}

\author{
Mostafa Sami M. Mostafa \\ Professor of Computer Science \\ Faculty of Computers and \\ Information \\ Helwan University
}

\begin{abstract}
Multi-core processor is viewed as the future production of microprocessor design. It is not only a solution for CPU speeds but also it decreases the power consumption, because many cores in a lower frequency collectively produce less heat dissipation than one core with their total frequency. From this point of view, Cloud computing will be mostly built on top of multi-core technologies. However, to fully take advantage of the computational capability and the other advantages of multi-cores, a lot of new techniques must be proposed and considered. In this research paper, a resourcescheduling algorithm along with a server consolidation algorithm is applied to multi-core processors. It is be shown by experimental results that adding cores to the processors in data centers increases the system performance, decreases the power consumption, along with other benefits.
\end{abstract}

\section{General Terms}

Cloud Computing, Scheduling, Multi-core processors, Cloudsim, Power Consumption.

\section{Keywords}

Cloud Computing, Scheduling, Multi-core processors, Cloudsim.

\section{INTRODUCTION}

Cloud computing is a technology that employs the internet and central remote servers to save data and applications. It allows users and businesses to use applications without installation; also to access their personal files from any place connected to the internet. This technology allows for much more efficient computing, by unifying storage, memory, processing, and bandwidth [1]. It refers to both the applications delivered as services over the Internet and the hardware and systems software in the datacenters offering those services [2]. Cloud computing is changing the way industries and organizations do their businesses in that dynamically scalable and virtualized resources are presented as a service over the Internet [3, 4]. Nowadays, the main concern in cloud computing is turning Green, that means using computing resources in an efficient and eco-friendly way [5]. Finding solutions for the resources allocation problem, (that leads to better resources utilization and energy efficient consumption), gains huge importance from scientific researchers. Using multi-core processors is also taken into considerations to improve expected results.

This paper is organized as follows, Section 2, introduces some related works. Section 3, defines multi-core processors, their benefits and their challenges. Section 4, shows how virtualization plays a key role in the Cloud. Section 5, contains results and their analysis. Finally, in Section 6, readers will find the research conclusion.

\section{RELATED WORK}

Sergio et al [6] addressed a multi-criteria non-pre-emptive energy aware scheduling problem for computational Grid systems. The main contributions of their work are listed as follows. i) the formulation of a new mathematical model for the energy consumption in multi-core High Computing (HC) Grid systems. ii) the description of a new multi-objective scheduling problem for HC Grid systems to minimize makespan and energy utilization. iii) the implementation and evaluation of twenty new fast bi-objective list scheduling heuristics for the problem. iv) the generation and declaration of new large problem instances. And v) a scalability analysis studying the usefulness of the best heuristics found when facing scenarios with different ratios between the number of tasks and the number of machines.

Viswanathan et al [7], presented a gang scheduling algorithm that satisfies the following characteristics under light load 1)Freedom from starvation, 2) Predictable and acceptable response, 3) Better processor utilization, 4) Simplicity. From the quality of service point of view, they have proved the importance of standard deviation in response time than the average response time. They presented a starvation free gang scheduling algorithm for multi-core processors with no process migration. For fairness, they proposed a new metric called bypass count, demonstrated its use in guaranteeing fairness by setting a bound on the number of bypasses possible over a waiting gang. The performance and the fairness of the proposed algorithm in comparison with the current best algorithm are verified through a simulation study.

Shohei et al [8], proposed a task-scheduling algorithm for a multi-core processor system that decreases the recovery time in case of a single fail stop failure of a multi-core processor. The presented algorithm is based on an existing checkpointing method. They assumed that the state is saved when nodes send results to the next node. They designed their algorithm as a parallel algorithm that achieves $\mathrm{O}(\boldsymbol{n})$ speedup where $\boldsymbol{n}$ is the number of processors.

Referring to the older research paper presented by the authors [9], the proposed algorithm was proved to be a scalable system that helps data centers using energy in an efficient way, by combining a resource allocation algorithm and a server consolidation one, its goal is to minimize the number of physical machines (PMs) used to execute all required tasks. This system was simulated using Cloudsim, but all physical machines are of single core processors. This research study is an enhancement for the earlier proposed system but using physical machines of multi-core processors. 


\section{MULTI-CORE PROCESSORS}

The microprocessor industry maintains to have enormous importance in the course of technological development ever since their coming to life in 1970s. Multi-core processors have been in reality since the past decade, but they got more importance later due to technology restrictions. Single-core processors are facing problems today, such as high throughput and long lasting battery life with high-energy consumption. Motivated by a performance hungry marketplace, microprocessors have always been planned to keep performance and cost in mind.

\subsection{Multi-core Definition}

"A Multi-core processor is typically a single processor which contains several cores on a chip". [10]

The cores are practical units made up of computation units and caches. These multiple cores on a single chip combine to replicate the performance of a single faster processor. The individual cores on a multi-core processor don't essentially run as fast as the highest performing single-core processors, but they get better overall performance by managing more tasks in parallel. The performance improvement can be evaluated by understanding the manner in which single core and multi-core processors are running programs.

Single core processors running different programs would assign time slice to execute one program and then assign different time slices for the rest of the programs. If one of the processes is taking longer time to be finished then all the rest of the processes start lagging behind. However, In the case of multi-core processors if you have multiple tasks that can be run in parallel at the same time, so a separate core will execute each of them in parallel, thus making improved performance [11].

\subsection{Benefits}

In multi-core technology, two or more CPUs are working together on the same chip. In this type of architecture, a single physical processor contains the core logic of two or more processors. These processors are packaged into a single integrated circuit $(\boldsymbol{I C})$. These single $\boldsymbol{I C}$ s are known as a die. Multi-core technology can also refer to many dies packaged together. This technology permits the system to execute more tasks with a better overall system performance. It also helps in decreasing the power consumption. Multi-core processors can be used in desktops, mobile personal computers (PCs), servers, and workstations. Therefore this technology is used to speed up the processing in a multitenant cloud environment. Multi-core architecture has become the recent trend of high performance processors, and various theoretical and case studies results illustrate that multi-core architecture is relative to the number of cores [12].

\subsection{Challenges}

The main issue is how efficiently the multi-core processors are used in the server clouds today, in order to get the real parallelism in terms of performance gain in a multitenant cloud environment using efficient programming [12].

Also the consolidation of a set of virtual machines on multicore systems, posed many questions that must be answered, such as:

- How to schedule a virtual machine across multiple cores, multiple processors, or even multiple clusters?

- How to move a virtual machine when slots of cores are available?

\section{VIRTUALIZATION}

In the server consolidation approach, virtualization technology plays an important key role. This technology with the popularity of multi-core processors have greatly assured and improved parallel processing; that is, a single multi-core processor often executes many tasks at the same time. This parallel processing practice at a glance appears to essentially increase performance and productivity. But the trade-off between this increase and the energy consumption should be carefully studied.

That is, load imbalance in those multi-core processors in particular is a major source of energy drainage. This fact has much produced many server consolidation studies [13].

\section{RESULTS AND COMPARISONS}

Offering a core to a single VM removes the potential software cache-based side channel, which is present when sharing an L1 cache. This may not look realistic, as many associate virtualization with maxing out resources on each physical server to run as many VMs on it as it can afford. However, it is believed that this counters to (i) the trend in computing towards multi-core, and (ii) the cloud computing model.

The trend in processors is to increase the number of cores on the chip with each generation (as opposed to using clock frequency as the main source of performance improvements). With each generation, the number of $\boldsymbol{V M} \mathbf{s}$ a server can support would increase with the number of cores. Also sharing of cores to hold up more $\boldsymbol{V M}$ s will not be necessary [14].

As presented in the early part of the authors' work, the system has been run using the Cloudsim package, using the same cases, but by allocating physical machines of dual-core processors.

\subsection{Experimental Results}

This section shows clearly the differences, in all cases, between using single-core and dual-core processors. Then three graphs will visually show how this modification enhanced the results especially for larger environments.

PMs' performance is equivalent to 1860 and 2660 MIPS, 4 MB of RAM, and $1 \mathbf{G B}$ of storage. Each $\boldsymbol{V M}$ requires one CPU core with 50, 100, 200, or 250 MIPS. RAM and storage resources for $\boldsymbol{V M} \mathrm{s}$ are minimized while they are out of research. Also all requests are of length equal to 450 Million Instruction $\mathbf{M I}$. The upper threshold used for CPU utilization is $85 \%$.

Results are compared from three different points of view, a) the energy consumption, b) number of VMs migrations and c) the mean time before a host status changed to idle.

They are represented in Tables from 1 to 5 .

Table 1. Case 1 consists of 10 PMs and $100 \mathrm{VMs}$

\begin{tabular}{|c|c|c|c|}
\hline & $\begin{array}{c}\text { Total simulation time: 900.10 sec } \\
\text { Proposed } \\
\text { System }\end{array}$ & $\begin{array}{c}\text { Energy } \\
\text { consumption }\end{array}$ & $\begin{array}{c}\text { Number of VM } \\
\text { migrations } \\
\text { before } \\
\text { a host status } \\
\text { changed to } \\
\text { idle } \\
\text { sec }\end{array}$ \\
\hline Single-core & 0.03 & 10 & 420.13 \\
\hline double-core & 0.02 & 4 & 390.12 \\
\hline
\end{tabular}


Table 2. Case 2 consists of 50 PMs and 500 VMs

\begin{tabular}{|c|c|c|c|}
\hline & $\begin{array}{c}\text { Total simulation time: 1200.10 sec } \\
\text { Proposed } \\
\text { System } \\
\text { consumption }\end{array}$ & $\begin{array}{c}\text { Number of VM } \\
\text { migrations } \\
\text { before } \\
\text { a host status } \\
\text { changed to } \\
\text { idle } \\
\text { sec }\end{array}$ \\
\hline Single-core & KWh & 38 & 480.13 \\
\hline double-core & 0.13 & 21 & 402.11 \\
\hline
\end{tabular}

Table 3. Case 3 consists of 100 PMs and 1000 VMs

\begin{tabular}{|c|c|c|c|}
\hline & Total simulation time: 1510.21 sec \\
\hline $\begin{array}{c}\text { Proposed } \\
\text { System }\end{array}$ & $\begin{array}{c}\text { Energy } \\
\text { consumption }\end{array}$ & $\begin{array}{c}\text { Number of VM } \\
\text { migrations } \\
\text { before } \\
\text { a host status } \\
\text { changed to } \\
\text { idle } \\
\text { sec }\end{array}$ \\
\hline Single-core & 0.76 & 138 & 304.24 \\
\hline double-core & 0.45 & 83 & 181.23 \\
\hline
\end{tabular}

Table 4. Case 4 consists of 500 PMs and 5000 VMs

\begin{tabular}{|c|c|c|c|}
\hline & $\begin{array}{c}\text { Total simulation time: 1900.10 sec } \\
\begin{array}{c}\text { Proposed } \\
\text { System } \\
\text { consumption }\end{array}\end{array}$ & $\begin{array}{c}\text { Number of VM } \\
\text { migrations }\end{array}$ & $\begin{array}{c}\text { Mean time } \\
\text { before } \\
\text { a host status } \\
\text { changed to } \\
\text { idle } \\
\text { sec }\end{array}$ \\
\hline Single-core & KWh & & 314.33 \\
\hline double-core & 1.5 & 215 & 215.31 \\
\hline
\end{tabular}

Table 5. Case 5 consists of 1000 PMs and 10000 VMs

\begin{tabular}{|c|c|c|c|}
\hline & Total simulation time: 1900.10 sec \\
\hline $\begin{array}{c}\text { Proposed } \\
\text { System }\end{array}$ & $\begin{array}{c}\text { Energy } \\
\text { consumption }\end{array}$ & $\begin{array}{c}\text { Number of } \\
\text { VM } \\
\text { migrations }\end{array}$ & $\begin{array}{c}\text { Mean time } \\
\text { before } \\
\text { a host status } \\
\text { changed to } \\
\text { idle } \\
\text { sec }\end{array}$ \\
\hline Single-core & 5.39 & 816 & 310.43 \\
\hline double-core & 3.07 & 425 & 218.01 \\
\hline
\end{tabular}

The visual representation for the above comparisons is shown in Figures 1-3

\subsection{Analysis}

From the results found, it's clearly shown that adding another core to the processors used, 1) increases the system performance in running more tasks in the same physical machine, also 2) decreases the power consumption and, 3) minimizes the number of virtual machine migrations. All these profits are so beneficial to organizations, especially to larger ones, while costs will be minimized with the same number of tasks executed, for private clouds or even for public clouds. While in private clouds, organizations will buy less physical but powerful multi-core machines, and for public clouds, providers' profit will increase due to the decrease of the power consumption.

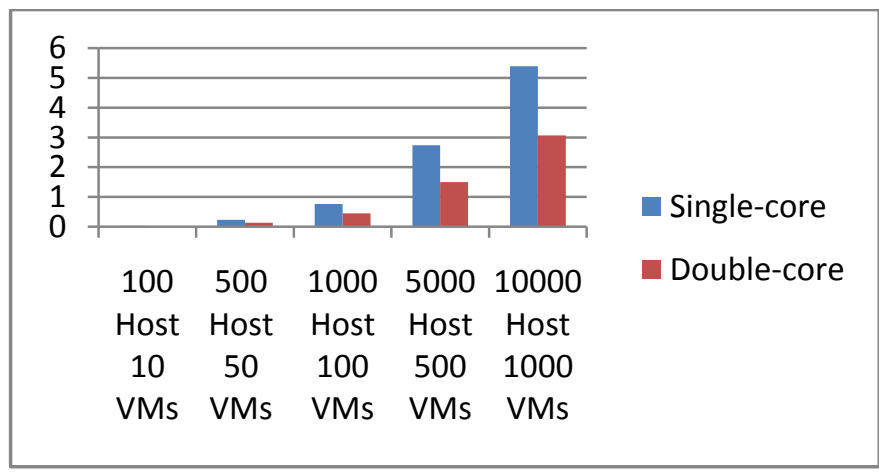

Fig 1: The energy consumption for the five cases, using single-core and dual-core processors

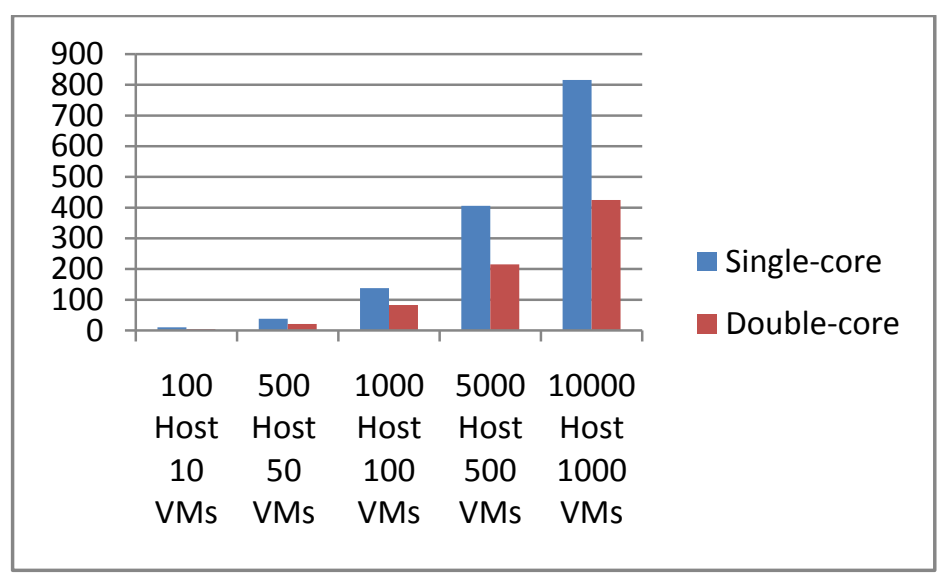

Fig 2: The number of migrations for the five cases, using single-core and dual-core processors

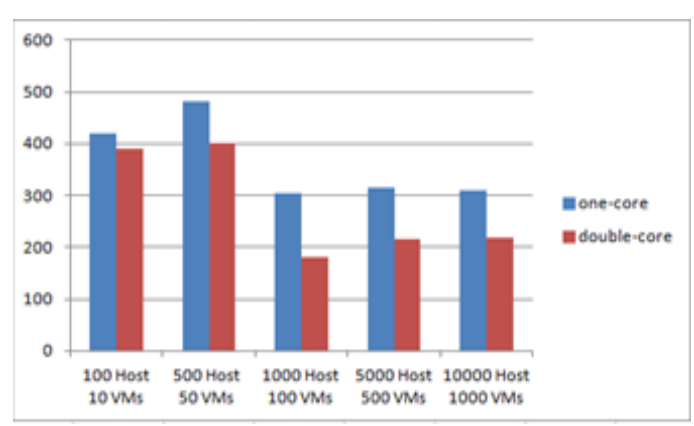

Fig 3: The mean time before a host shut down for the five cases, using single-core and dual-core processors

\section{CONCLUSION}

Multi-core advantages can be summarized as follows: 1) A chip multiprocessor consists of simple-to-design cores, 2) Simple design leads to more power efficiency and, 3) High system performance in parallel applications where many threads need to run simultaneously [15]. All these benefits make the multi-core technology a better choice for Cloud Computing and it is expected that all future computer systems will be based on multi-core processors [7]. 


\section{REFERENCES}

[1] Yuping Xing and Yongzhao Zhan, Y. Zhang, Virtualization and Cloud Computing, (Ed.):Future Wireless Networks and Information Systems, LNEE 143, pp. 305-312. springerlink.com () Springer-Verlag Berlin Heidelberg 2012

[2] Michael Armbrust, Armando Fox, Rean Griffith, Anthony D. Joseph, Randy Katz, Andy Konwinski, Gunho Lee, David Patterson, Ariel Rabkin, Ion Stoica, and Matei Zaharia, Above the Clouds: A Berkeley View of Cloud Computing, UC Berkeley Reliable Adaptive Distributed Systems Laboratory, http://radlab.cs.berkeley.edu/, February 2009

[3] Madhukara Phatak and Kamalesh.V.N, On Cloud Computing Deployment Architecture, JSS Academy of Technical Education, Bangalore, 2010 International Conference on Advances in ICT for Emerging Regions (ICTer), IEEE, 2010

[4] T.Swathi et al, Research Article Virtualization In Cloud Computing, International Journal of Computer Science and Mobile Computing, Vol.3 Issue.5, May- 2014, IJCSMC, 2014

[5] S.V.S.S. Lakshmi, Ms. I Sri Lalita Sarwani, M.Nalini Tuveera, A Study On Green Computing: The Future Computing And Eco-Friendly Technology, International Journal of Engineering Research and Applications (IJERA) ISSN: 2248-9622 www.ijera.com Vol. 2, Issue4, pp.1282-1285, 2012

[6] Sergio Nesmachnow, Bernabé Dorronsoro, Johnatan E. Pecero, Pascal Bouvry, Energy-Aware Scheduling on Multicore Heterogeneous Grid Computing Systems, J Grid Computing, 2013
[7] Viswanathan Manickam and Alex Aravind, A Fair and Efficient Gang Scheduling Algorithm for Multicore Processors, Springer-Verlag, Berlin, Heidelberg, 2012

[8] Shohei Gotoda, Minoru Ito, Naoki Shibata, Task scheduling algorithm for multicore processor system for minimizing recovery time in case of single node fault, IEEE International Symposium on Cluster Computing and the Grid, CCGrid, 2012

[9] Mostafa Sami, M. Haggag, Dina Salem, Resource Allocation and Server Consolidation Algorithms for Green Computing, International Journal of Scientific \& Engineering Research, Vol 6, Issue 12, December 2015

[10] Lizhe Wang, Jie Tao, Gregor von Laszewski, Holger Marten, Multicores in Cloud Computing: Research Challenges for Applications, Journal of Computers, Vol 5, No 6, 2010

[11] Balaji Venu, Multi-core processors-An overview, Department of Electrical Engineering and Electronics, University of Liverpool, Liverpool, UK

[12] K. Chandrasekaran, Essentials of Cloud Computing,

[13] Young Choon Lee-Albert Y. Zomaya, Energy efficient utilization of resources in cloud computing systems, J Supercomputer 60:268-280, 2012

[14] Eric Keller Jakub Szefer Jennifer Rexford Ruby B. Lee, NoHype: Virtualized Cloud Infrastructure without the Virtualization Princeton University, Saint-Malo, France, ISCA'10, 19-23, June 2010

[15] Attia KM et al., Dynamic Power Management Techniques In Multi-Core Architectures: A Survey Study, Ain Shams Eng J, 2015, http://dx.doi.org/10.1016/j.asej.2015.08.010 